\title{
e-Tümlenmiş Modüllerin Torsiyon Teorisine Göre Genelleştirilmiş Bir Versiyonu
}

\author{
Esra ÖZTÜRK SÖZEN ${ }^{1 *}$ \\ ${ }^{1}$ Sinop Üniversitesi, Fen Edebiyat Fakültesi, Matematik Bölümü
}

Geliş / Received: 12/09/2019, Kabul / Accepted: 21/02/2020

$\ddot{O} z$

$\mathrm{Bu}$ çalışmanın amacı $e$-tümlenmiş modüllerin torsiyon-teorik bir genelleştirmesini elde etmektir. Bunun için öncelikle bir modülün $\tau_{e}$-küçük alt modülleri tanımlanarak temel özelliklerine değinildi. Ardından $\tau_{e}$-tümlenmiş modül ve $\tau_{e}$-oyuk modül kavramlarına yer verilerek bunlara ilişkin temel teorik özellikler irdelendi.

Anahtar Kelimeler: Kalıtsal torsiyon teorisi, $\tau_{e}$-küçük alt modül, $\tau_{e}$-tümlenmiş modül, $\tau_{e}$-oyuk modül

\section{A Generalized Version of $e$-Supplemented Modules Relative to a Torsion Theory}

\begin{abstract}
The objective of this aim to obtain a torsion theoretic analogue of e-supplemented modules. For this, firstly we define $\tau_{e}$-submodule of a module and give basic properties of this concept. After that, $\tau_{e}$-supplemented modules and $\tau_{e}$-hollow modules are introduced and investigated some fundamental properties of these modules.
\end{abstract}

Keywords: Hereditary torsion theory, $\tau_{e}$-small submodule, $\tau_{e}$-supplemented module, $\tau_{e}$-hollow module.

\section{Giriş}

Öncelikli olarak belirtelim ki, Bu çalışmada $R, M$ ve $\tau$ notasyonları ile sirasiyla birimli bir halka, üniter bir $R$-modül ve kalitsal bir torsiyon teorisi temsil ediliyor olacaktır. $M$ modülünün $N$ (öz) alt modülü (' $N<M$ ') ' $N \leq M$ ' ile gösterilir. Bir $M$ modülünün $N$ alt modülü için $N+K=M$ koşulu yalnızca $K=$ $M$ olduğunda sağlanıyorsa $N$ ye $M$ nin bir küçük alt modülü denir ve ' $N \ll M$ ' ile gösterilir. Buna karşı1lk, $N$ alt modülünün $M$

\footnotetext{
*Sorumlu Yazar: esozen@ sinop.edu.tr
}

nin $\{0\}$ alt modülü haricindeki diğer tüm alt modülleri ile arakesiti s1firdan farklı ise $N$ ye $M$ nin büyük alt modülü denir ve ' $N \unlhd M$ ' ile gösterilir.

Son yıllarda, küçük alt modül kavramının genelleştirmesini içeren kaydadeğer çalışmaların varlı̆̆ dikkatleri çekmektedir (Zhou, 2000; Zhou and Zhang, 2011). Bu kavramlardan en göze çarpanlarını $\delta$-küçük alt modüller ve $e$-küçük alt modüller (Koşar vd. (2015) bu kavramı literatüre $g$-küçük alt modül olarak atfetmiştir.) olarak belirtebiliriz. 
Tümlenmiş modül, projektif örtü ve dolayısıyla yarı mükemmel modül kavramlarının verilmesinde anahtar rolü oynayan küçük alt modüller bu yönüyle modül ve halka teorinin her sürecinde merkezi bir konuma sahiptir. Dolayısıyla bunların biraz önce değindiğimiz genelleştirilmiş versiyonları ise torsiyon teorisine göre yeni varyasyonlarına erişmek için oldukça değerlidir.

$N \leq M$ ve $K \unlhd M$ olmak üzere $N+K=M$ ifadesi yalnızca $K=M$ olduğunda gerçekleniyor ise $N$ ye $M$ nin $e$-küçük alt modülü denir ve ' $N \ll_{e} M$ ' ile gösterilir (Zhou ve Zhang, 2011). $N \leq M$ için $N+K=$ $M$ ve $N \cap K \ll_{(e)} K$ olacak şekilde bir $K \leq M$ alt modülü var ise $K$ ya $N$ nin $M$ de bir $(e-)$ tümleyeni denir. $M$ modülünün her alt modülü $M$ de bir tümleyene sahip ise $M$ ye $(e-)$ tümlenmiş modül denir. (Burada bahsi geçen $(e-)$ tümleyen ve $(e-)$ tümlenmiş modül kavramlarını Koşar vd. (2015) sirasıyla ( $g$-) tümleyen ve $(g-)$ tümlenmiş modül olarak irdelemiştir. Küçük alt modül, büyük alt modül, $\delta$-küçük alt modül, $e$-küçük alt modül gibi temel bilgilerin detaylarına erişmek için sirasiyla Clark vd. (2006), Zhou (2000), Quynh vd. (2013) tarafindan yapilan çalışmalar okuyucuya refere edilebilir.

$\mathrm{Bu}$ çalışmanın amacı $e$-tümlenmiş modüller için bilinen kavramların ve sonuçların torsiyon teorik versiyonlarını elde etmektir. Buna geçmeden önce Materyal ve metod bölümünde torsiyon teorisinde kullanılan $\tau$ torsiyon modül, $\tau$-torsiyon serbest modül, $\tau$ yoğun, $\tau$-pür ve $\tau$-büyük alt modül gibi bilinmesi gereken temel kavramlara yer verilmiştir. Ayrıca çalışma içerisinde $\tau_{e}$-oyuk modüller tanımlanarak temel özellikleri irdelenmiştir. $\mathrm{Bu}$ çalışmanın sonunda $e$ tümlenmiş modüllerden daha genel olan $\tau_{e^{-}}$ tümlenmiş modüller literatüre kazandırılmıştır. Bununla birlikte, $e$ tümlenmiş ( $g$ - tümlenmiş) modüllerin bilinen tüm genelleştirilmiş versiyonlarının torsiyon teorik olarak irdelenmesini sağlayabilecek çalışmaların zemini oluşturulmuştur.

Sonuç olarak aşağıdaki hiyerarşiden bahsetmek mümkündür:

tümlenmiş
modül $\Rightarrow \begin{gathered}\text { O-tümlenmiş } \\ \text { modül }\end{gathered} \Rightarrow \begin{gathered}\text { e-tümlenmiş } \\ \text { modül }\end{gathered} \Rightarrow \begin{gathered}\text { t-e-tümlenmiş } \\ \text { modül }\end{gathered}$

\section{Materyal ve Metot}

Tanım 2.1: $R$-mod da bir $\tau$-torsiyon teorisi aşağıdaki koşulları gerçekleyen $\tau=(\mathcal{T}, \mathcal{F})$ sınıflarının ikilisidir.

1. $\mathcal{T} \cap \mathcal{F}=\{0\}$.

2. $T \rightarrow M \rightarrow 0$ dizisi tam ve $T \in \mathcal{T}$ ise $M \in \mathcal{T}$ dir.

3. $0 \rightarrow M \rightarrow F$ dizisi tam ve $F \in \mathcal{F}$ ise $M \in \mathcal{F}$ dir.

4. Her $M$ modülü için $0 \rightarrow T \rightarrow M \rightarrow$ $F \rightarrow 0$ dizisi tam olacak şekilde $T \in$ $\mathcal{T}$ ve $F \in \mathcal{F}$ modülleri vardır (Bland, 1998).

Tanım 2.2: $M \in \mathcal{T}$ ise $M$ ye $\tau$-torsiyon modül; $M \in \mathcal{F}$ ise $M$ ye $\tau$-serbest torsiyon modül denir (Bland, 1998).

Tanım 2.3: $\tau=(\mathcal{T}, \mathcal{F}) \tau$-torsiyon teorisinde $\tau(M)=\sum\{N \leq M \mid N \in \tau\}$ olmak üzere

$\mathcal{T}=\{M \in R-\bmod \mid \tau(M)=M\} ;$

$\tau$-torsiyon modüllerin sinıfıdır.

$\mathcal{F}=\{M \in R-\bmod \mid \tau(M)=0\} ;$

$\tau$-serbest torsiyon modüllerin sinıfıdır (Bland, 1998).

Tanım 2.4: $\mathcal{T}$ sınıfı alt modüller altında kapalı ise yani içerdiği $\tau$-torsiyon modüllerin her alt 
modülü de $\tau$-torsiyon ise $\tau$ ya kalitsal torsiyon teorisi denir (Bland, 1998).

Tanım 2.5: $\mathcal{F}$ sınıfı homomorfik görüntüler altında kapalı ise $\tau$ ya eşkalıtsal torsiyon teorisi denir (Bland, 1998).

Önerme 2.6: $\mathcal{T}$ sınıfı homomorfik görüntüler, direkt toplamlar ve genişlemeler altında kapalıdır (Bland, 1998).

Önerme 2.7: $\mathcal{F}$ sinıfı alt modüller, direkt çarpımlar ve genişlemeler altında kapalıdır (Bland, 1998).

Tanım 2.8: $M$ bir modül ve $N \leq M$ olsun. $M / N$ bölüm modülü $\tau$-torsiyon ise $N$ ye $M$ nin $\tau$-yoğun alt modülü denir ve " $N \leq_{\tau-d} M "$ ile gösterilir (Bland, 1998).

Tanım 2.9: $M$ bir modül ve $N \leq M$ olsun. $M / N$ bölüm modülü $\tau$-serbest torsiyon ise $N$ ye $M$ nin $\tau$-pür alt modülü denir ve $" N \leq_{\tau-p} M "$ ile gösterilir. Ayrıca $M$ modülünün tüm $\tau$-yoğun alt modüllerinin kümesi " $\mathcal{D}_{\tau}(M)^{\prime \prime}$; tüm $\tau$-pür alt modüllerinin kümesi " $\mathcal{P}_{\tau}(M)^{\prime \prime}$ ile gösterilir. Buna göre $M$, $\tau(M) \in \mathcal{P}_{\tau}(M)$ olduğu kolaylıkla görülebilir (Bland, 1998).

Tanım 2.10: $M$ bir modül ve $N \leq M$ olsun. $N \unlhd M$ ve $N \leq_{\tau-d} M$ ise $N$ ye $M$ nin $\tau$-büyük alt modülü denir ve " $N \unlhd_{\tau} M^{\prime \prime}$ ile gösterilir (Bland, 1998).

Her $\tau$-büyük alt modülün büyük alt modül olduğu açıktır. Ve tanımları gereği $\tau$-büyük alt modülün büyük alt modülün bir genelleştirilmiş versiyonu değil özelleştirilmişi olduğuna ulaşılabilir.

$\mathrm{Bu}$ bölümde verilen tüm tanım ve diğer bilgilerin detaylı formu için Bland (1998) a ait kaynak incelenebilir.

\section{Bulgular}

Tanım 3.1: $M$ bir modül ve $N \leq M$ olsun. $K \unlhd_{\tau} M$ için $N+K=M$ ifadesi yalnızca $K=M$ olduğunda gerçekleniyorsa $N$ ye $M$ nin $\tau_{e}$-küçük alt modül denir ve ${ }^{\prime \prime} N \ll_{\tau_{e}} M^{\prime \prime}$ ile gösterilir.

Bir modülün her $e$-küçük alt modülü $\tau_{e^{-}}$ küçüktür. $\tau$-torsiyon modüller için tersi de söylenebilir.

Şimdi $\tau_{e}$-küçük alt modüllerin özelliklerini aşağıdaki önerme ile verelim.

Önerme 3.2: $M$ bir modül olsun.

1. $K, L, N \leq M$ ve $\mathrm{K} \leq N$ olsun.

a) $N \ll_{\tau_{e}} M$ ise $K \ll_{\tau_{e}} M$ ve $N / K \ll_{\tau_{e}} M / K$ dir.

b) $N+L \ll_{\tau_{e}} M \Leftrightarrow N \ll_{\tau_{e}} M$ ve $L \ll_{\tau_{e}} M$ dir.

2. $\tau_{e}$-küçük alt modüllerin homomorfik görüntüsü de $\tau_{e}$-küçük alt modüldür.

3. $K_{1} \leq M_{1} \leq M, K_{2} \leq M_{2} \leq M$ ve $M=M_{1} \oplus M_{2}$ olsun. Bu durumda, $K_{1} \oplus K_{2} \ll_{\tau_{e}} M_{1} \oplus M_{2} \Leftrightarrow K_{1} \ll_{\tau_{e}} M_{1}$ ve $K_{2} \ll_{\tau_{e}} M_{2}$ dir.

4. $K \leq N \leq M, K \ll_{\tau_{e}} M$ ve $N, M$ nin direkt toplam terimi ise $K \ll_{\tau_{e}} N$ dir.

Tanım 3.3: $M$ bir modül ve $U, V \leq M$ olsun. $M=U+V$ ve bir $T \unlhd_{\tau} V$ için $M=U+T$ olması $T=V$ olmasinı gerektiriyorsa $V$ ye $U$ nun $M$ de $\tau_{e}$-tümleyeni denir. Eğer $M$ nin her alt modülü $\tau_{e}$-tümleyene sahipse $M$ ye $\tau_{e^{-}}$ tümlenmiş modül denir.

$E$-tümlenmiş her modül $\tau_{e}$-tümlenmiştir.

Lemma 3.4: $M$ bir modül ve $U, V \leq M$ olsun. $U$ alt modülünün $M$ de bir $V \tau_{e}$-tümleyene 
sahip olması için gerek ve yeter koşul $U+$ $V=M$ ve $U \cap V \ll_{\tau_{e}} V$ olmasidir.

İspat: $(\Longrightarrow): V, U$ nun $M$ de $\tau_{e}$-tümleyeni olsun. $\mathrm{Bu}$ durumda $U+V=M$ olduğu açıtır. $U \cap V \ll_{\tau_{e}} V$ olduğunu göstermek yeterlidir. Bir $T \unlhd_{\tau} V$ için $U \cap V+T=V$ olsun. Buradan $M=U+V=U+T$ olup hipotez gereği $T \unlhd_{\tau} V$ için $T=V$ bulunur.

$(\Leftarrow): U+V=M$ ve $U \cap V \ll_{\tau_{e}} V$ olsun. $T \unlhd_{\tau} V$ için $M=U+T$ ise modüler kuralından $V=(U+T) \cap V=T+(U \cap V)$ olup $U \cap V \ll_{\tau_{e}} V$ ve $T \unlhd_{\tau} V$ olduğundan $T=V$ bulunur.

Lemma 3.5: $M$ bir modül ve $M_{1}, U \leq M$ olsun. $M_{1} \tau_{e}$-tümlenmiş ve $M_{1}+U, M$ de bir

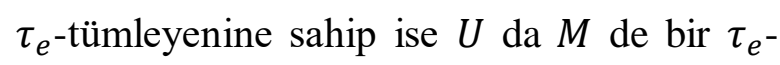
tümleyenine sahiptir.

İspat: $X, M_{1}+U$ nun $M$ de bir $\tau_{e}$-tümleyeni olsun. $\mathrm{Bu}$ durumda $\left(M_{1}+U\right)+X=M$ ve $\left(M_{1}+U\right) \cap X \ll_{\tau_{e}} X$ dir. $M_{1} \tau_{e}$-tümlenmiş olduğundan $M_{1} \cap(U+X), M_{1}$ de bir $Y \tau_{e^{-}}$ tümleyeni sahiptir. $\mathrm{Bu}$ durumda $\left[M_{1} \cap(U+\right.$ $X)]+Y=M_{1} \quad$ ve $\quad\left[M_{1} \cap(U+X)\right] \cap$ $Y \ll_{\tau_{e}} Y$ dir. Buradan $M=\left(M_{1}+U\right)+X=$ $\left[M_{1} \cap(U+X)\right]+Y+U+X=U+X+Y$ ve $\quad U \cap(X+Y) \leq[X \cap(U+Y)]+[Y \cap$ $(U+X)] \leq\left[X \cap\left(M_{1}+U\right)\right]+\left[\left(Y \cap M_{1}\right) \cap\right.$ $(U+X)] \ll_{\tau_{e}} X+Y$

elde edilir. Böylece $X+Y$ nin $U$ nun $M$ de bir $\tau_{e}$-tümleyeni olduğu görülür.

Yukarıdaki Lemma kullanılarak sonlu sayıda

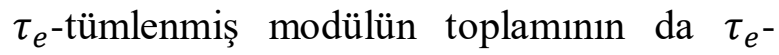
tümlenmiş olduğu söylenebilir.

Teorem 3.6: $M_{1}$ ve $M_{2} \quad \tau_{e}$-tümlenmiş modüller ise $M_{1}+M_{2}$ de $\tau_{e}$-tümlenmiştir.

İspat: Yukarıdaki Lemma gereği açıktır.
Sonuç 3.7: Sonlu sayıda $\tau_{e}$-tümlenmiş modülün toplamı da $\tau_{e}$-tümlenmiştir.

Lemma 3.8: $M$ bir modül ve $X \leq U \leq M$ olsun. $V, U$ nun $M$ de $\tau_{e}$-tümleyeni ise $(V+X) /_{X}$ de $U / X$ in $M / X$ de bir $\tau_{e^{-}}$ tümleyenidir.

İspat: $V, U$ nun $M$ de $\tau_{e}$-tümleyeni olduğundan $U+V=M$ ve $U \cap V \ll_{\tau_{e}} V$ yazılabilir. $\quad \mathrm{Bu}$ durumda $[(U \cap V)+X] /_{X} \ll_{\tau_{e}}(V+X) /_{X}$ dir. Ayrica $M=U+V \quad$ olduğundan $M / X=(U+V) /_{X}=U / X+(V+X) / X \quad$ ve $U / X \cap((V+X) / X)=$ $[(U \cap V)+X] /_{X} \ll_{\tau_{e}}(V+X) /_{X}$ olduğundan $(V+X) /_{X}, U / X$ in $M / X$ de bir $\tau_{e}$-tümleyenidir.

Teorem 3.9: $M \tau_{e}$-tümlenmiş bir modül ise her faktör modülü de $\tau_{e}$-tümlenmiştir.

Sonuç 3.10: $\tau_{e}$-tümlenmiş her modülün homomorfik görüntüsü de $\tau_{e}$-tümlenmiştir.

Tanım 3.11: Bir $M$ modülü için $\operatorname{Rad}_{\tau_{e}}(M)=$ $\bigcap\left\{N \leq_{\max } M \mid N \unlhd_{\tau} M\right\} \operatorname{dir}$.

Lemma 3.12: Bir $M$ modülü için $\operatorname{Rad}_{\tau_{e}}(M)=\sum_{L \ll \tau_{e} M} L$ dir.

İspat: $L, M$ nin herhangi bir $\tau_{e}$-küçük alt modülü olsun. $L$ nin maksimal ve aynı zamanda $\tau$-büyük olan $M$ nin her alt modülü tarafından kapsandığ 1 gösterilmelidir. Bunun için aksine, $M$ nin bir $T$ maksimal, $\tau$-büyük alt modülü için $L \nsubseteq T$ olduğu kabul edilirse $T$ nin maksimalliği gereği $L+T=M$ olur. $L \ll_{\tau_{e}} M$ ve $T \unlhd_{\tau} M$ olduğundan $T=M$ çelişkisi elde edilir. Öyleyse, $L, M$ nin her maksimal $\tau$-büyük alt modülünde kapsanır. 
Yani $\sum_{L \ll \tau_{e} M} L \subseteq \operatorname{Rad}_{\tau_{e}}(M)$ dir. Tersine her $x \in \operatorname{Rad}_{\tau_{e}}(M) \quad$ için $\quad R x \ll_{\tau_{e}} M \quad$ olduğu gösterilirse $\quad R x \subseteq \sum_{L \ll_{\tau_{e}} M} L \quad$ olacağından $\operatorname{Rad}_{\tau_{e}}(M) \subseteq \sum_{L \ll \tau_{e} M} L$ elde edilir. Bunun için, $R x \ll_{\tau_{e}} M$ olmadığ 1 varsayılırsa $\emptyset \neq$ $\Omega=\left\{T \unlhd_{\tau} M \mid x \notin T, R x+T=M\right\}$

kümesinin her zincirinin kapsama bağıntısına göre bir en küçük üst sınırı mevcuttur. Öyleyse Zorn Lemması gereği bir $K \in \Omega$ maksimal elemanı mevcuttur. Dolayısıyla $K, M$ nin $\tau$-büyük maksimal bir alt modülü olup $\operatorname{Rad}_{\tau_{e}}(M) \subseteq K$ olup $x \notin K$ olduğundan $x \notin \operatorname{Rad}_{\tau_{e}}(M)$ çelişkisi elde edilir. $\mathrm{Bu}$ durumda, her $\quad x \in \operatorname{Rad}_{\tau_{e}}(M) \quad$ için $R x \ll_{\tau_{e}} M$ dir. Buradan $x \in R x \subseteq \sum_{L \ll_{\tau_{e}} M} L$ olup $\operatorname{Rad}_{\tau_{e}}(M) \subseteq \sum_{L \ll \tau_{e} M} L$ bulunur.

Sonuç 3.13: $\operatorname{Bir} M$ modülü için $\operatorname{Rad}(M) \subseteq$ $\delta(M) \subseteq \operatorname{Rad}_{e}(M) \subseteq \operatorname{Rad}_{\tau_{e}}(M) \operatorname{dir}$.

Lemma 3.14: Bir $M$ modülü için aşağıdaki ifadeler sağlanır.

1. Her $m \in \operatorname{Rad}_{\tau_{e}}(M)$ için $R m \ll_{\tau_{e}} M$ dir.

2. $N \leq M$ ise $\operatorname{Rad}_{\tau_{e}}(N) \leq \operatorname{Rad}_{\tau_{e}}(M)$ dir.

3. $f: M \rightarrow N$ modül homomorfizmas1 olmak üzere $f\left(\operatorname{Rad}_{\tau_{e}}(M)\right) \leq \operatorname{Rad}_{\tau_{e}}(N)$ dir.

4. $M=\bigoplus_{i \epsilon I} M_{i} \quad$ ise $\quad \operatorname{Rad}_{\tau_{e}}(M)=$ $\bigoplus_{i \in I} \operatorname{Rad}\left(M_{i}\right) \operatorname{dir}$.

İspat: Kaynak (Wisbauer 1991, Bölüm 21.6) incelenerek benzer şekilde ispat yapılabilir.

Tanım 3.15: Bir $M$ modülünün her öz alt modülü $\tau_{e}$-küçük ise $M$ ye $\tau_{e}$-oyuk modül denir. Bir $R$ halkası $R$-modül olarak $\tau_{e}$-oyuk ise $R$ ye $\tau_{e}$-oyuk halka denir.

Yarı basit modüllerin ve $e$-oyuk modüllerin $\tau_{e}$-oyuk olduğu açıktır. Ayrıca $\tau$-torsiyon bir modülün her $\tau_{e}$-oyuk alt modülünün de $e$ oyuk olduğu açıktır. Diğer taraftan $\mathbb{Z}_{6}$, $\mathbb{Z}$ - modülü $\tau_{e}$-oyuk olup oyuk olmayan bir modül örneğidir.

Önerme 3.16: $\operatorname{Bir} M$ modülünün $\tau_{e^{- \text {oyuk }}}$ olması için gerek ve yeter koşul $M$ nin her öz $\tau$-büyük alt modülünün $M$ de küçük olmasıdır. İspat: $(\Longrightarrow): N, M$ nin öz $\tau$-büyük alt modülü olmak üzere $N$ nin $M$ de küçük olmadığını kabul edelim. Bu durumda Bir $W<M$ öz alt modülü için $W+N=M$ dir. $N \unlhd_{\tau} M$ ve $M$ $\tau_{e}$-oyuk olduğundan $W \ll_{\tau_{e}} M$ olup $N=M$ çelişkisi elde edilir. $M \tau_{e}$-oyuk iken her öz $\tau$ büyük alt modülü $M$ de küçüktür.

( $\Longleftarrow$ ): $M$ nin her öz $\tau$-büyük alt modülünün $M$ de küçük olsun. $N, M$ nin herhangi bir öz alt modülü olmak üzere $W \unlhd_{\tau} M$ için $N+W=$ $M$ olsun. $W \neq M$ ise hipotez gereği $W \ll M$ olduğundan $N=M$ çelişkisi elde edilir. $\mathrm{Bu}$ durumda $W=M \operatorname{olup} N \ll_{\tau_{e}} M$ dir.

Önerme 3.17: Bir $M$ modülü için $\operatorname{Rad}_{\tau_{e}}(M) \neq M$ olsun. Bu durumda aşağıdaki ifadeler denktir:

1. $M$ modülü $\tau_{e}$-oyuktur.

2. $M$ modülü lokaldir.

3. $M$ modülü oyuktur.

İspat: $(1 \Rightarrow 2): M$ modülü $\tau_{e}$-oyuk ve $T$ de $M$ nin herhangi bir öz alt modülü olsun. $\mathrm{Bu}$ durumda $T \ll_{\tau_{e}} M \quad$ olduğundan $T \leq$ $\operatorname{Rad}_{\tau_{e}}(M)$ dir. Hipotez gereği $\operatorname{Rad}_{\tau_{e}}(M) \neq$ $M$ olduğundan, $\operatorname{Rad}_{\tau_{e}}(M), M$ nin her öz alt modülünü içeren en büyük öz alt modülü olur. Öyleyse $M$ modülü lokaldir.

$(2 \Rightarrow 3) \quad$ ve $\quad(3 \Rightarrow 1) \quad$ önermelerinin doğruluğu açıktır.

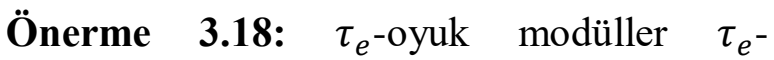
tümlenmiştir. 
İspat: $\tau_{e}$-oyuk modül tanımı gereği $M$ modülünün kendisi her alt modülünün $\tau_{e^{-}}$ tümleyeni olur. Böylelikle iddia doğrulanır.

Önerme 3.19: $M$ bir öz $\tau$-büyük alt modüle sahip sonlu üretilmiş bir modül olsun. Bu durumda $M$ nin her öz $\tau$-büyük alt modülünü içeren bir maksimal $\tau$-büyük alt modülü vardır.

İspat: $K, M$ nin herhangi bir öz $\tau$-büyük alt modülü olsun. $M$ sonlu üretilmiş olduğundan her öz alt modülü içeren bir maksimal alt modül mevcuttur. Özel olarak $K$ yı da içeren bir $P<M$ maksimal alt modülü vardır. $K \leq$ $P<M$ ve $K \unlhd_{\tau} M$ olduğundan $P \unlhd_{\tau} M$ olup istenen elde edilir.

Önerme 3.20: $M, \operatorname{Rad}_{\tau_{e}}(M) \neq M$ olacak şekilde bir modül olsun. $M$ nin her öz $\tau$-büyük alt modülü $M$ nin bir maksimal $\tau$-büyük alt modülün tarafindan kapsanırsa $\operatorname{Rad}_{\tau_{e}}(M) \ll_{\tau_{e}} M$ dir.

\section{Sonuç}

Sonuç ve Tartışma: Modül ve halka teoride bilinen kavramlarin genellemelerinin ve torsiyon teorik kurgulamalarının yapılmasının temeli, bir modülün belli bir özelliğini tüm alt modülleri üzerinde araştırmak yerine, onun $\tau$-yoğun ya da $\tau$-pür alt modüllerine kendimizi kisitlayarak araştırmaktır. $\mathrm{Bu}$ amaçla çalışmamızda bir modülün $\tau$-büyük alt modüllerinden (ki aynı zamanda t-yoğun alt modüllerdir) faydalanarak tanımladığımız $\tau_{e}$-tümlenmiş modüller ile $e$-tümlenmiş modüllerin yeni bir genelleştirmesi elde edilmiş ve temel özellikleri irdelenmiştir.

Öneriler: Bu çalışma ile birlikte $e$-tümlenmiş modüllerin kalitsal bir torsiyon teorisine göre yeni bir genelleştirilmesi elde edilmiştir. $\mathrm{Bu}$ yönüyle aslında bilinen tüm diğer tümlenmiş modül çeşitlendirmelerinin, üzerinde modellenebileceği yeni bir modül türü okuyucuya sunulmuştur. $\mathrm{Bu}$ bağlamda $\tau_{e^{-}}$ tümlenmiş modüllerin genelleştirilmesi başlı̆̆ 1 altında pek çok varyasyonel çalışma hazırlanabilir.

Teşekkür: Bu çalışmanın değerlendirilmesi ve geliştirilmesi aşamasında değerli katkılarını esirgemeyen hakem hocalarımıza teşekkürü bir borç bilirim.

\section{Kaynaklar}

Bland, P. E. 1998. 'Topics in Torsion Theory', Mathematical Research, 103, Wiley-VCH, Berlin.

Charalambides, S. and Clark, J. 2007. 'CS Modules Relative to a Torsion Theory, Mediterr. J. Math., 4, 291-308.

Clark, J., Lomp, C., Vanaja, N., and Wisbauer, R. 2006. Lifting modules: Supplements and Projectivity in Module Theory, Birkhauser, Verlag, Basel.

Koşan M.T. 2007. $\delta$-lifting and $\delta$-supplemented Modules, Algebra Colloquim, 14(1), 53 - 60.

Koşar, B., Nebiyev, C. and Sökmez, N. 2015. ' $G$-supplemented Modules', Ukrainian Mathematical Journal, 67(6): 861-864.

Quynh, T. C. and Tin, P. H. 2013. 'Some Properties of $e$-supplemented and $e$-lifting Modules', Vietnam Journal of Mathematics, 41(3), 303-312.

Wisbauer, R. 1991. Foundations of Module and Ring Theory, Gordon and Breach.

Zhou, Y. 2000. Generalizations of Perfect, Semiperfect, and semiregular rings, Algebra Colloq., 7(3), 305-318. 
$e$-Tümlenmiş Modüllerin Torsiyon Teorisine Göre Genelleştirilmiş Bir Versiyonu

Zhou, D. X. and Zhang, X. R. 2011. 'Smallessential submodules and Morita duality' Southeast Asian Bulletin of Mathematics, 35(6), 1051-1062. 